\title{
The Analysis of Psychopathic Traits and Behavioral Problems Relationships in Italian Adolescents
}

\author{
Luca Rossi \\ Niccolò Cusano University of Rome, Italy \\ E-mail: luca.rossi@unicusano.it \\ Margot Zanetti \\ Niccolò Cusano University of Rome, Italy \\ E-mail: margot.zanetti@unicusano.it
}

Received: June 12, $2021 \quad$ Accepted: June 20, $2020 \quad$ Published: June 23, 2021

doi:10.5296/jet.v8i1.18748

URL: http://dx.doi.org/10.5296/jet.v8i1.18748

\begin{abstract}
In this article we analysed the association between psychopathic traits manifested at early age and behavioral problems in adolescents with an extension of correspondence analysis. The used technique allows to verify the relationship between row and column variables in a two-way contingency table. The data are obtained submitting to a sample of 689 high school students two questionnaires: The Inventory of Callous-Unemotional Traits (ICU) and The Strengths and Difficulties Questionnaire (SDQ)). Founding has an important pedagogical impact. The educational professionals, who spend most of the day with the kids, hardly can identify the Callous-unemotional traits but, at same time, could identify easily behavioral problems allowing the implementation of early treatments or the use of pedagogical strategies for young people that could have a high risk of psychopathic traits.
\end{abstract}

Keywords: Correspondence analysis, callous-unemotional traits, behavioral problems, psychopathic traits

\section{Introduction}

The last few years have seen a significant increase in literature and research dealing with psychopathological disorders in childhood and adolescence (Gritti et al., 2014). Such studies are related to understanding the origin of the disorders, how to treat them, how to manage them and, last but not least, their strong connection with the development of a serious criminal career. Hence, their correct understanding could prevent adult deviance and criminal acts and contribute to the well- being of the individual. However, if on the one side such disorders have been thoroughly investigated in psychology and psychiatry, on the other side similar studies have not been conducted in depth from a pedagogical perspective. Pedagogy is the key to the 


\section{Macrothink}

management of problematic behaviours and to the education (or re-education) of individuals. Moreover, learning is one of the principal pedagogical interests thus, incorporating psychological and neuroscientific findings about behavioral disorders, could help in didactical and educational strategies. Children and adolescents spend most of their time in school interacting with teachers and education professionals and this is the context in which conduct (and learning) difficulties are more evident. The complications that teachers must face are nature multiple and comprehend the duty to deal with, not only the individual with conduct problems, but also their peers and the relationship between them. To have an instrument that helps to catch the symptoms or alarming behaviors is fundamental. Surely, not all disruptive behaviours must be considered pathological. It is appropriate to believe that sometimes a pattern of behaviours is considered pathological merely for differencing from the norm and to lighten parents and teachers from their responsibilities.

Many epidemiological studies, furthermore, conducted by Kessler (2005), Merikangas (2010) and Polancyzyk (2015) have showed that a large chunk of adolescents (13-25\%) might meet the criteria for a mental disorder during their lifetime. In particular, behavior problems such as oppositional-defiant disorder, conduct disorder, attention-deficit and hyperactivity disorder and emotional problems such as anxiety disorders and depression are frequently diagnosed among youths. The behavior problems are somehow easier identifiable by an expert teacher and there are easy screening tools that can be used for detecting youths who are at high risk for developing behavioral and emotional complications. The Strengths and Difficulties Questionnaire (SDQ) (Goodman, 1997 and 1999) is a well-validated brief emotional and behavioral screening questionnaire for detecting possible mental health problems in children and young people that is able to rank, basing on cutoff points, classify subjects as normal, borderline, or abnormal. In literature there are various studies that validated this test (Goodman, 2001; Lundh, Wangby-Lundh, and Bjarehed, 2008; Law and Wolpert, 2014; Hall, Guo, Valentine, Groom, Daley, Sayal, Hollis, 2019).

Correspondence analysis is a descriptive technique that affords to define the relationship between qualitative variables, measured in nominal and ordinal scales (Beh 2004). This technique, as well as the principal component analysis and factor analysis for quantitative variables, simplifies the interpretation of the data even if although some information is missing. The use of particular graphs allows the researcher for easy, intuitive reasoning on the relationships between the analyzed categories of variables (Kilon and Dębkowska, 2013). The correspondence analysis is a method to decompose the Pearson chi-squared statistics by defining a system with a small number of dimensions, in which the deviations from the expected values are presented. (Bendixen 2003). The aim is to reduce the number of analyzed space dimensions by choosing such a low-dimensional subspace in which the chi-square distances between points are shown with the best accuracy (Greenacre et Hastie, 1987). Interpretation of the correspondence biplot allows researchers to find the diversity within the analyzed variables profiles and to highlight the data that give a major contribution to the total inertia.

Here our focus is on studying the association among the psychopathic traits on emotion 
recognition (callousness, uncaring and unemotional) measured using the Inventory of CallousUnemotional Traits (ICU) and the behavioral problems of adolescents (normal, borderline and abnormal) measured using Strengths and Difficulties Questionnaire (SDQ). The aim is not to make diagnosis but to detect the presence of $\mathrm{CU}$ traits and behavioral problems that could be related to it, to be able to build effective learning and educational plans. We propose the use of correspondence analysis to visualize not simply the association between row categories or column categories but above all the correlation between row and column profiles.

This paper is divided into further five section. A focus on psychopathic traits in section 2 . Section 3 provides a theoretical description of use of Correspondence analysis. In section 4 are showed the results applying the model to the data. Some final remarks are made in section 5.

\section{The callous-unemotional traits}

Psychopathy is a complex phenomenon associate to personality disorder characterized by affective, interpersonal, and behavioral characteristics. Recent studies (Levy at al.,2015; Moore at al., 2017) have highlighted that psychopathy at a young age, manifests with aggressive and violent behaviours and it tends to be relatively stable throughout life. Researchers have called these conduct disorders "callous- unemotional traits" (CU) and the theoretical framework from which they were thought was initially derived from the concept of psychopathy in adults (Frick and Ellis, 1999). Individuals with CU traits can be also characterized, by other un-emotional reactions such as the lack of guilt and a limited displays of emotion, and, similarly to the adult construct of psychopathy, elements of interpersonal style, such as a failure to show empathy and exploitation of others to advance personal interests. CU traits, in general, identify a subgroup of individuals with distinctive clinical and neurocognitive characteristics (Blair et al., 2014). Assuming all these information, it follows that CU traits are considered strong predictors of the development of a serious deviant and criminal career (Bergstrøm and Farrington, 2018). The Inventory of Callous-Unemotional Traits (ICU) is one of the most widely used measurements to assess CU traits (Frick, 2004).

Starting from these remarks could be reasonable to think that teachers or education professionals, although they spend most of the day with the kids, hardly can identify the CU traits in students whether they do not use aggressiveness or unproportioned reactions. They could be more evident when students seem insensitive to different form of punishment and do not show remorse for their bad actions. The possibility to associate these traits to behavioral problems is fundamental to early detection of the psychopathy, allowing the implementation of early treatments or the use of pedagogical strategies for young people who have a high risk of severe conduct disorders.

To understand the real extent of the problem and to investigate the presence of the CU traits even in the very young Italian population and to assess the correlation with the behavioral problems, the tests for the detection of callous-unemotional (Frick, 2004) and the well-tested Strengths and Difficulties Questionnaire (Vostanis,2006), were administered in two Italian high schools. The questionnaires have been submitted to a sample of 689 high school students 14 to 19 years of age in Veneto and Lazio (two Italian regions of North and South Italy 
respectively). The sample was diverse in regards to parental educational level but representative of families in the school districts.

In this work, for a more complete study, we explored also the correlations of every single CU trait with the two types of problematic behaviours considered in literature: the externalizing behaviours (Bishop, Okagbue and Odukoya, 2020) like aggressiveness, impulsivity, and control problems and internalizing behaviours (Liu, Chen and Lewis, 2011) like withdrawal, anxiety, and depression. The presence of even one of the two types of problematic leads to less acceptance by peers and greater social difficulties in the individual. This research has been conducted also to highlight possible behavioral differences between gender, geographical origin and family situation.

This paper is, obviously, the first step of a wider project without diagnostic purpose but with the aim of detecting the problem and possible strategies to deal with it.

\section{Methodology}

\section{Notation}

Let $\mathrm{A}\left(n_{i j}\right)$ be a two way-contingency table that cross-classifies $\mathrm{N}$ units into I row categories and $\mathrm{J}$ column categories. Let the cell probability define by $p_{i j}=P\left(I=x_{i} \cap J=y_{j}\right)=\frac{n_{i j}}{N}$, where $x_{i}$ is the $i$-th category of $\mathrm{I}$ and $y_{j}$ is the $j$-th category of $\mathrm{J}$ so that $\sum_{i=1}^{I} \sum_{j=1}^{J} \frac{n_{i j}}{N}=\sum_{i=1}^{I} \sum_{j=1}^{J} p_{i j}=1$. Define the $i$-th row marginal probability $p_{i *}=\sum_{j=1}^{J} p_{i j}$ and the $j$-th column marginal probability $p_{* j}=\sum_{i=1}^{I} p_{i j}$ (Agresti 2002).

\section{Correspondence analysis}

Correspondence analysis, like the other analytic techniques, determines the strength of association between two or more variables in a contingency table.

In a two-way contingency table, a model for complete independence between rows and column variables (Roy and Kastembaum, 1956) is

$$
\frac{p_{i j}}{p_{i *} * p_{* j}}=1
$$

In consequence a model for partial dependence can be expressed using a multiplicative coefficient $\alpha_{i j}$ :

$$
\frac{p_{i j}}{p_{i *} * p_{* j}}=\alpha_{i j}
$$

For the model of complete independence $\alpha_{i j}$ is equal to 1 for all $i=i \ldots . I$ and $j=1 \ldots . J$.

A classical test to verify whether observations consisting of measures on two variables, expressed in contingency table are independent of each other is Pearson chi-squared statistic: 


$$
\chi^{2}=N * \sum_{i=1}^{I} \sum_{j=1}^{J} \frac{p_{i j}-p_{i *} * p_{* j}}{p_{i *} * p_{* j}}
$$

By considering equation 1, Pearson chi-squared statistic can be expressed by:

$$
\begin{gathered}
\chi^{2}=N * \sum_{i=1}^{I} \sum_{j=1}^{J} \frac{\left(p_{i j}-p_{i *} * p_{* j}\right)^{2}}{p_{i *} * p_{* j}}=N * \sum_{i=1}^{I} \sum_{j=1}^{J} \frac{\left(\alpha_{i j} * p_{i *} * p_{* j}-p_{i *} * p_{* j}\right)^{2}}{p_{i *} * p_{* j}}= \\
=N * \sum_{i=1}^{I} \sum_{j=1}^{J} p_{i *} * p_{* j} *\left(\alpha_{i j}-1\right)^{2}
\end{gathered}
$$

and has a Pearson chi-squared distribution with $(I-1)(J-1)$ degrees of freedom. Of course, a small Pearson chi-squared statistic will be achieved when each $\alpha_{i j} \approx 1$ and is consistent with the hypothesis of independence. Pearson chi-squared statistic increases with $\mathrm{N}$, to overcome this problem in correspondence analysis we can consider the total inertia of contingency table $\frac{\chi^{2}}{N}$.

By decomposing the total inertia is possible identify information that describes the association between variables. In correspondence analysis the decomposition normally used is Singular value decomposition (SVD). Eckart and Young in 1936 showed that Pearson ratio can be decomposed into components by

$$
\alpha_{i j}=\sum_{k=0}^{K} \beta_{i k} \lambda_{k} \delta_{j k}
$$

where $K=(\max (I, J)-1)$ is the number of dimensions required to plot the association between row and column vectors, $\beta_{i k}$ and $\delta_{j k}$ are respectively the $k$-th component of $i$-th row score and $j$-th column score. The elements $\lambda_{k}$ are positive and real and are the first $K$ singular values arranged in way that:

$$
\lambda_{0}=1 \geq \lambda_{1} \geq \cdots \geq \lambda_{K} \geq 0
$$

The singular values can be computed trough the equation:

$$
\lambda_{k}=\sum_{i=1}^{I} \sum_{j=1}^{J} \beta_{i k} p_{i j} \delta_{j k}
$$

and the two singular vectors have the property that $\sum_{i=1}^{I} \beta_{i k} \beta_{i k^{\prime}} p_{i *}$ and $\sum_{j=1}^{J} \delta_{j k} \delta_{j k^{\prime}} p_{* j}$ are both equal to 1 whether $k=k^{i}$ and 0 otherwise.

The equation 2 , considering the property can be written as: 


$$
\alpha_{i j}=1+\sum_{k=1}^{K} \beta_{i k} \lambda_{k} \delta_{j k}
$$

By considering the previous equation and the orthogonally properties of $\beta_{i k}$ and $\delta_{j k}$, the total inertia can be written in terms of singular values:

$$
\frac{\chi^{2}}{N}=\sum_{k=1}^{K} \lambda_{k}^{2}
$$

A graphical representation of association between row and column vectors can be given plotting the set of singular vectors $\beta_{i k}$ and $\delta_{j k}$ as coordinates into the $k$-th dimension of a correspondence plot. In this plotting system the vectors do not consider the strength of the relationship between row and column vectors and for this scope is better to use a system of standard co-ordinates (Greenacre, 1984) in which the two singular vectors can be defined as:

$$
\begin{aligned}
& f_{i k}=\beta_{i k} \lambda_{k} \\
& g_{j k}=\delta_{j k} \lambda_{k}
\end{aligned}
$$

and have the property that $\sum_{i=1}^{I} f_{i k} f_{i k^{\prime}} p_{i *}$ and $\sum_{j=1}^{J} g_{j k} g_{j k^{\prime}} p_{* j}$ are both equal to $\lambda_{k}^{2}$ whether $k=k^{i}$ and 0 otherwise. Instead of each axis having unit inertias, the $k$-th principal axis has inertia equal to $\lambda_{k}^{2}$. The total inertia can be written as:

$$
\frac{\chi^{2}}{N}=\sum_{i=1}^{I} \sum_{k=1}^{K} p_{i *} f_{i k}^{2}
$$

or

$$
\frac{\chi^{2}}{N}=\sum_{j=1}^{J} \sum_{k=1}^{K} p_{* j} g_{j k}^{2}
$$

The equations 4 and 5 highlight that co-ordinates close to the origin give a small contribute to total inertia and do not contribute much to the variation of the data instead co-ordinates far from the origin give a major contribute. The distance between the $i$-th row or $j$-th column profile respectively from the origin can be computed by considering the Euclidean distance $\left(d^{2}\right)$ :

$$
\begin{aligned}
& d_{I}^{2}(i, 0)=\sum_{j=1}^{J} \frac{1}{p_{* j}}\left(\frac{p_{i j}}{p_{i *}}-p_{* j}\right)^{2}=\sum_{k=1}^{K}\left(\sum_{j=1}^{J} p_{* j} \delta_{j k}{ }^{2}\right) \beta_{i k}{ }^{2} \lambda_{k}{ }^{2}=\sum_{k=1}^{K} f_{i k}^{2} \\
& d_{J}^{2}(j, 0)=\sum_{i=1}^{I} \frac{1}{p_{j *}}\left(\frac{p_{i j}}{p_{* j}}-p_{i *}\right)^{2}=\sum_{k=1}^{K}\left(\sum_{i=1}^{I} p_{i *} \beta_{i k}{ }^{2}\right) \delta_{j k}{ }^{2} \lambda_{k}{ }^{2}=\sum_{\substack{k=1 \\
\text { jet.macrothink.org }}}^{K} g_{j k}^{2}
\end{aligned}
$$


The equations 6 and 7 clearly show that points far from the origin indicate a deviation from complete independence unlike points close to the origin highlight that respectively row or column frequencies of the contingency table fit the independence hypothesis well.

Another advantage of correspondence analysis is the possibility to graphically establish whether two profiles of the same variable are similar or not. The squared distance between two row profiles $i$ and $i^{\prime}$ written in terms of $f_{i k}$ and $f_{i^{\prime} k^{2}}$ is given by:

$$
d_{I}^{2}\left(i, i^{\prime}\right)=\sum_{j=1}^{J} \frac{1}{p_{* j}}\left(\frac{p_{i j}}{p_{i *}}-\frac{p_{i^{\prime} j}}{p_{i^{\prime} *}}\right)^{2}=\sum_{k=1}^{K}\left(f_{i k}-f_{i^{\prime} k}\right)^{2}
$$

Similarly, the Euclidean distance between two column profiles $j$ and $j^{\prime}$ written in terms of $g_{j k}$ and $g_{j^{\prime}}$ is given by:

$$
d_{I}^{2}\left(j, j^{\prime}\right)=\sum_{j=1}^{J} \frac{1}{p_{i *}}\left(\frac{p_{i j}}{p_{* j}}-\frac{p_{i j^{\prime}}}{p_{* j^{\prime}}}\right)^{2}=\sum_{k=1}^{K}\left(g_{j k}-g_{j^{\prime} k}\right)^{2}
$$

These results highlight that whether, in a correspondence plot, two row or column profiles are positioned closely to one another, then are similar else are different.

In this work the correspondence analysis is used to measure the correlation between a row and column profile. For this purpose, it is necessary to use a scaling displayed (the asymmetric biplot) in which the weighting of each point is important in determining the contributions made by each point to the solution. This approach is based on the scalar products between row and column vectors, which depend on the lengths of the vectors and the angles between them rather than their interpoint distances. The scope is that to find row points $x_{i}$ and column points $y_{j}$ such that the scalar products between the row and column vectors approximate the corresponding elements of the table as closely as possible. Using the equation proposed by Greenacre et Hastie, in 1987, the point plotted on the graph $\left(s_{i j}\right)$ are the sum of a scalar product in a low-dimensional subspace and a residual "error" term:

$$
s_{i j}=\sum_{k=1}^{K} x_{i k} y_{j k}+e_{i j}
$$

The biplot is fitted by minimizing the least square errors.

To appreciate the linkage between the correspondence analysis and the biplot, it is necessary to consider the equation (3), that expresses the original data in function of coordinates, and to multiply each term for the respective row and column masses:

$$
\frac{n_{i j}}{N}=r_{i} c_{i}\left(1+\sum_{k=1}^{K} \beta_{i k} \lambda_{k} \delta_{j k}\right)
$$




\section{MlMacrothink}

The mass of each row $r_{i}$ or column $c_{i}$ reflects its importance in the sample and it is the proportion of this row or column in the total of the table respectively.

The previous equation can be arranged so that the right hand-side is in the form of a scalar product:

$$
\frac{n_{i j}}{N r_{i} c_{i}}-1=\sum_{k=1}^{K} f_{i k} \delta_{j k}
$$

This shows that row asymmetric map, is an approximate biplot of the values on the left-hand side of the equation. The ratios $\frac{n_{i j}}{N r_{i} c_{i}}$ are called contingency ratios and values closer to 1 imply that the scalar product equal to zero and thus row and column profiles are approximately independent.

By the equation (8), for the construction of the graph, it is necessary the definition of the axes, i.e. to decide whether to analyze the contributions of rows against columns or vice versa. In case the contribution of rows to the axes has to be interpreted, the row points are represented in standard coordinates $\left(f_{i k}\right)$ and the column points in ordinal scores $\left(\delta_{j k}\right)$ multiplied by the mass. Similarly, in case in which the contribution of columns to the axes has to be interpreted, the column points are represented in standard coordinates $\left(g_{j k}\right)$ and the row points in ordinal scores $\left(\beta_{i k}\right)$ multiplied by the mass. For easy evaluation, all row and column scores are drawn using a vector that joins the origin of axis to its position using the Euclidean distance. These vectors in such a plotting system allow both to visualize the association between row or column categories (vectors that contribute very little to the solution, are close to the center of the biplot and are relatively unimportant to the interpretation, vectors far from origin, instead, give a major contribute to the total inertia) and to take in consideration the strength of the relationship between the rows and column along the axis, i.e. whether the angle between two arrows is acute, then there is a strong association between the corresponding row and column. The asymmetric map can be quite unsatisfactory when the total inertia is small, because the profiles represented in standard coordinated are concentrated into a small space at the center of the map, while the ordinal scores are very far out.

\section{Tests taken into consideration}

For this part of the research, the tests taken into consideration are the Inventory CallousUnemotional (ICU) and the Strengths and Difficulties Questionnaire (SDQ).

The SDQ is a 25-item screening instrument designed to evaluate social, emotional, and behavioral functioning in youth. It is made up of 4 clinical domains plus one: a) hyperactivityinattention; b) emotional symptoms; c) peer-relationship problems; d) conduct problems; e) prosocial behaviour. Each item is rated on a 3-point Likert scale. The psychometric properties of the SDQ have been verified in different cultural and clinical contexts and were found to be suitable (Vostanis, 2006).

The Inventory of Callous-Unemotional Traits (ICU) is a copyright protected 24-item 


\section{1) Macrothink

questionnaire, each of which is rated on a four-point scale $(0=$ not true to $3=$ very true), aimed at providing a complete assessment of callous and unemotional traits. These traits have proven to be relevant for defining a distinct subgroup group of antisocial and aggressive youth. The ICU has three subscales: Callousness, Uncaring, and Unemotional. It has been developed to be auto-administered or to be filled by persons near to the young under assessment (e.g. Teachers, parents). In this work the Youth Self- Report (Ciucci, Baroncelli et al., 2014) and all the three factors $(3 \mathrm{~F})$ have been used. The number factors assessed ranges from two to three, with varying content (Benesh et al., 2014; Henry et al., 2016). We decided to look for correlations of callousness, uncaring, and unemotional factors. Some scholars suggested to use two-factor (2F) models, usually including callousness and uncaring factors, ignoring the unemotional factor (Moore et al., 2017). The reason of this omission is that unemotional features are considered as not useful for detecting psychopathic traits or externalizing problems. We decided to keep also the evaluations of the items 2 and 10 of the ICU, because, even if, as underlid by Ciucci and Baroncelli (2013), present a lower correlation in the assessment of callous-unemotional traits.

\section{Case study}

In this section, we investigate the relationship between the psychopathic traits (callousness, uncaring and unemotional) measured using the Inventory of Callous-Unemotional Traits (ICU) and the behavioral problems of adolescents (normal, borderline and abnormal) measured using Strengths and Difficulties Questionnaire (SDQ). The investigation of data is carried out applying two-way correspondence analysis. The two-way contingency table (Table1) summarizes the number of students according to a particular trait of ICU (no_trait, callousness, uncaring and unemotional) and the potential diagnosis of SDQ (normal, borderline and abnormal).

Table 1 ICU/SDQ

\begin{tabular}{|l|l|l|l|l|}
\hline \multicolumn{2}{|c|}{} & \multicolumn{2}{l}{ SDQ } & \multicolumn{2}{l|}{} \\
\cline { 2 - 6 } & Normal & Borderline & Abnormal \\
\hline \multirow{4}{*}{ ICU } & No_trait & 218 & 64 & 46 \\
\cline { 2 - 6 } & callousness & 134 & 60 & 72 \\
\cline { 2 - 6 } & uncaring & 107 & 44 & 61 \\
\cline { 2 - 6 } & unemotional & 25 & 13 & 15 \\
\hline
\end{tabular}

The association between row and column variables is statistically significant, with Pearson's chi squared statistic equal to 28.071 on 6 degrees of freedom and p-value equal to $9.11 \mathrm{e}^{-05}$ and so a complete study using the correspondence analysis is able. All the following figures have 
been built considering the asymmetric biplot, where the callous-unemotional traits are plotted in standard coordinates (red vectors) and the behavioral problems in in ordinal scores (blue vectors) and are drawn using a vector that joins the origin of axis to its position. Whether the angle between two arrows is acute, then there is a strong association between the corresponding row and column. A first general correlation highlights that (figure 1):

a) individuals with prevalence of callousness have a normal SDQ;

b) young people with the prevalence of the uncaring trait result borderline in the SDQ;

c) subjects with a predominance of the unemotional dimension have and abnormal SDQ.

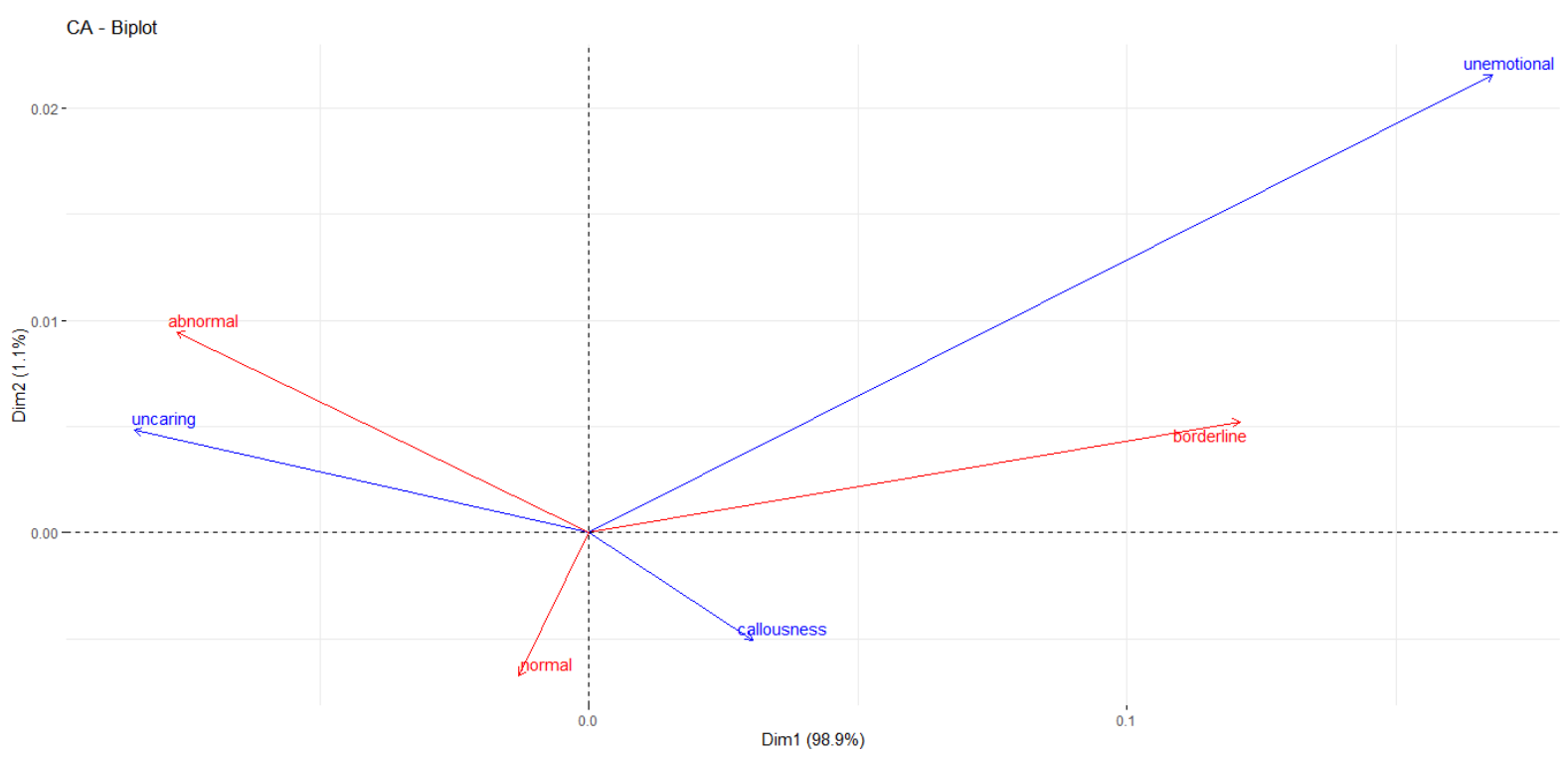

Figure 1. The asimmetric biplot using students with $\mathrm{CU}$ traits

In tables 2 and 3 are showed the associations obtained applying correspondence analysis considering gender and geographical area of students respectively.

Table 2. Gender influence in ICU and SDQ association

\begin{tabular}{|l|l|l|l|l|}
\hline \multicolumn{2}{|c|}{} & \multicolumn{2}{l|}{ SDQ } & \multicolumn{2}{l|}{} \\
\hline \multirow{2}{|l|}{} & Total & Women & Men \\
\hline \multirow{3}{*}{ ICU } & callousness & Normal & Normal & Normal \\
\cline { 2 - 6 } & uncaring & Borderline & Borderline & Borderline \\
\cline { 2 - 5 } & unemotional & Abnormal & Abnormal & Abnormal \\
\hline
\end{tabular}


Table 3. Geographical area influence in ICU and SDQ association

\begin{tabular}{|c|c|c|c|c|}
\hline & & \multicolumn{3}{|l|}{ SDQ } \\
\hline & & Total & North & South \\
\hline \multirow{3}{*}{ ICU } & callousness & Normal & Normal & Normal \\
\hline & uncaring & Borderline & Borderline & Borderline \\
\hline & unemotional & Abnormal & Abnormal & Abnormal \\
\hline
\end{tabular}

The results highlight that there is no gender or geographical influence in the association between the psychopathic traits and behavioral problems.

The variables that seem to affect the correlation are familiar situation (figure 4) (divorced/separated parents or united family) and the use of drugs (figure 5). The individuals with a separated family unit showed all the CU traits related to borderline or abnormal SDQ results.

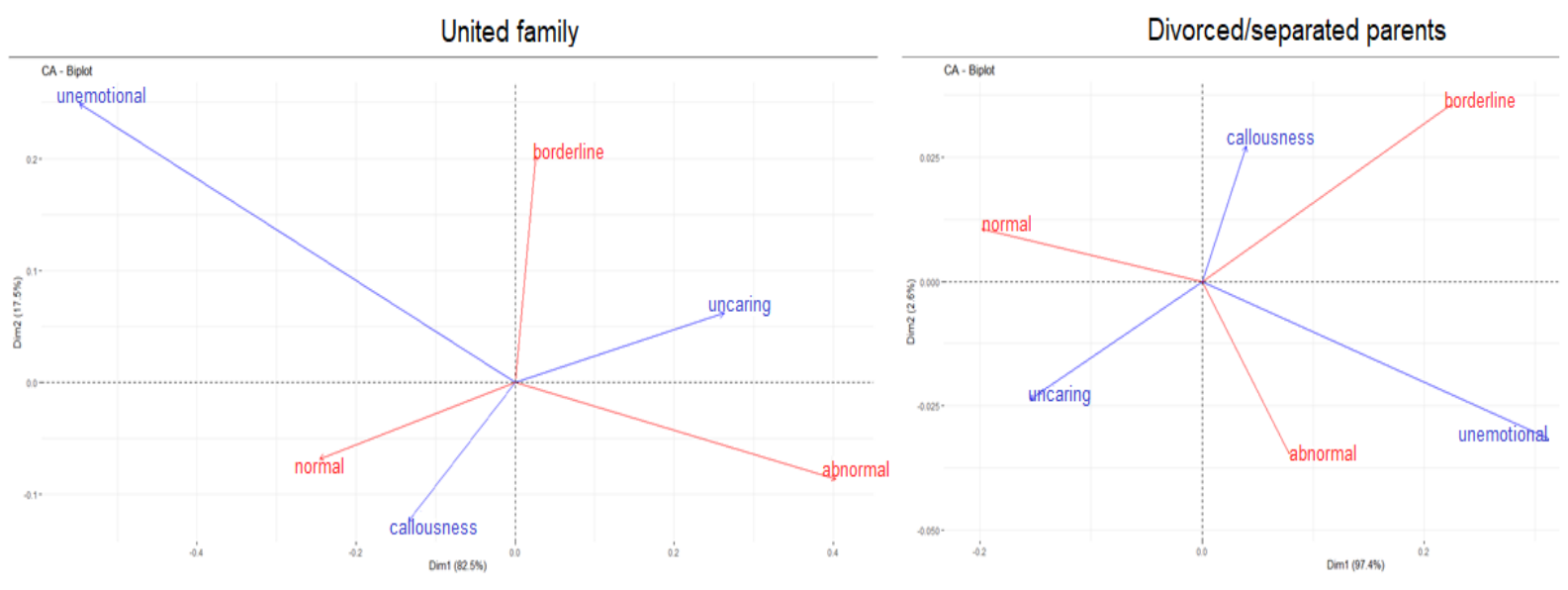

\section{Figure 4. The asimmetric biplot of familiar situation}

Precisely: individuals with the prevalence of callousness showed a borderline SDQ and those with a dominance of the uncaring and unemotional traits result abnormal.

Individuals who declared to use drugs, in presence of CU traits showed a different correlation with behavioral problems different from the association highlights in students who do not use drugs: subjects with callousness dominance result related to the abnormal dimension of the SDQ, those with a predominance of the uncaring or unemotional trait end up in the borderline dimension. 


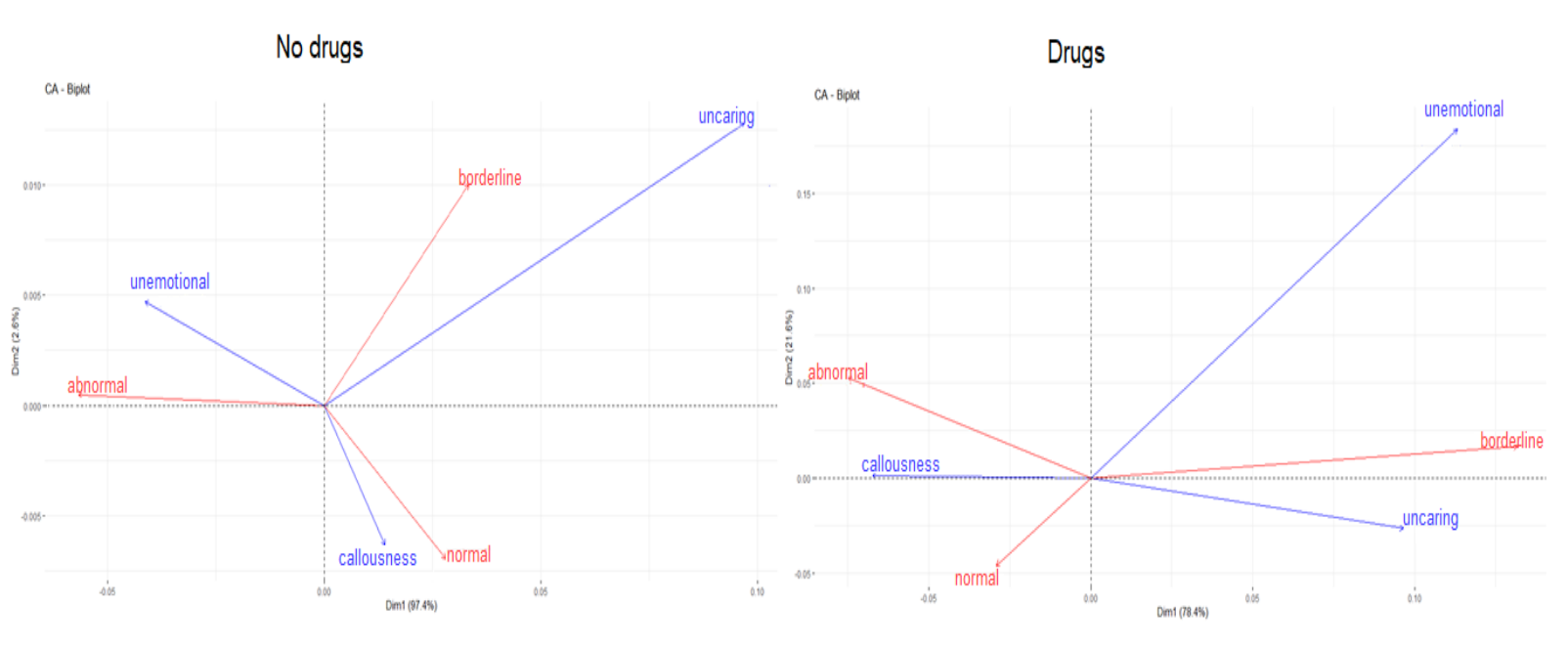

Figure 5. The asimmetric biplot of drug use

As already mentioned, in this paper, it is taken also in consideration the relationship between the externalizing scale of SDQ and CU traits.

Most individuals with CU traits get an SDQ that shows externalizing problems. More in detail, taking into account each trait it has been observed that the uncaring trait results in an abnormal SDQ while the unemotional gives a borderline score (figure 7).

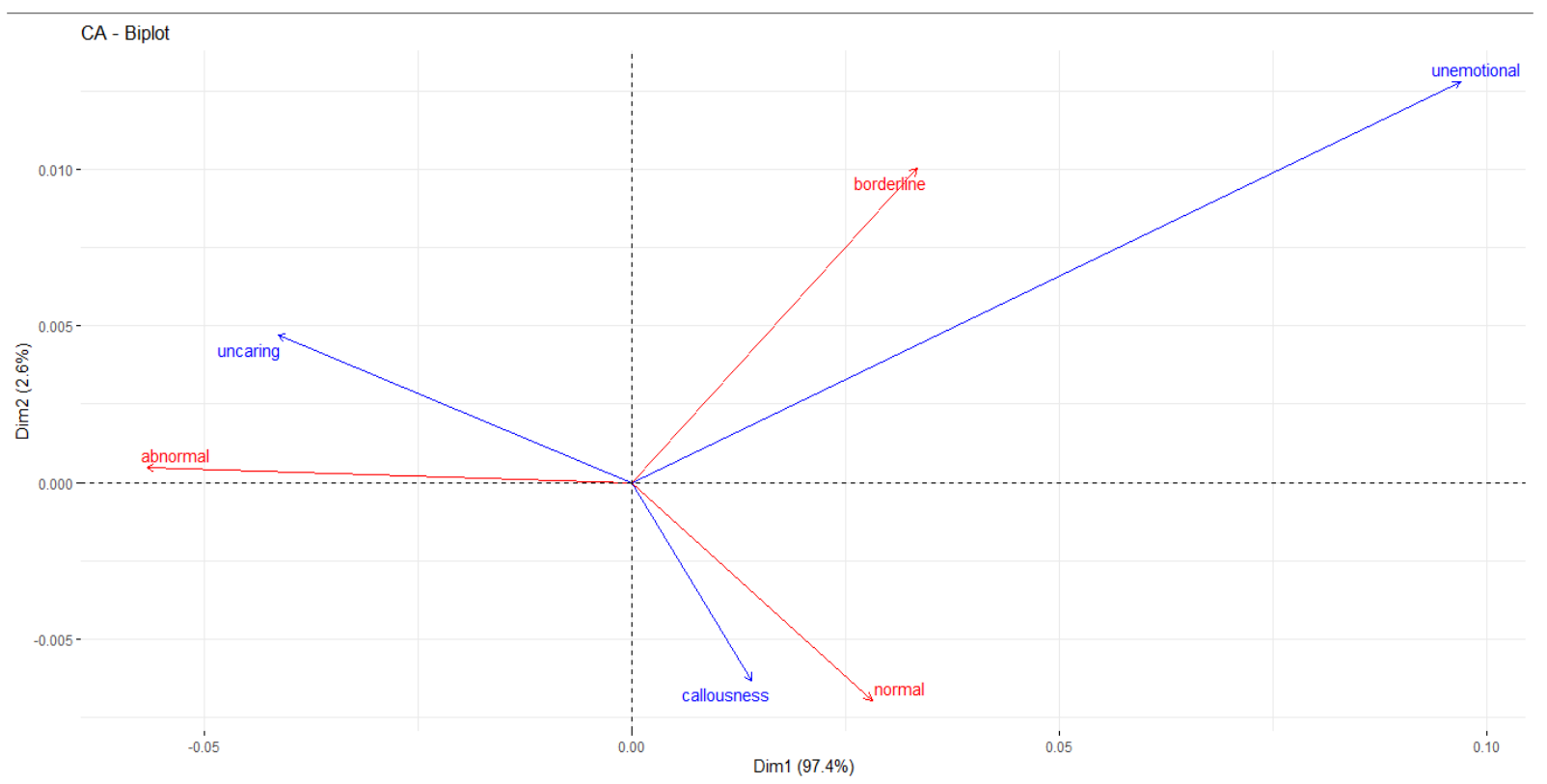

Figure 7. The asimmetric biplot considering the externalizing SDQ using students with CU traits

The externalizing problems are exacerbated by the family variable, by the use of drugs and unlike the complete scale of the SDQ also by gender variable. The tables 4,5 and 6 show the influence of variables mentioned above in the association between ICU traits and externalizing 


\section{Macrothink

SDQ.

Table 4. Drugs influence in ICU and externalizing SDQ association

\begin{tabular}{|l|l|l|l|l|l|}
\hline \multicolumn{2}{|l|}{} & & \multicolumn{2}{|l|}{ Externalizing SDQ } & \\
\hline \multicolumn{3}{|l|}{} & Total & No drugs & Drugs \\
\hline \multirow{3}{*}{ ICU } & callousness & Normal & Normal & Abnormal \\
\cline { 2 - 6 } & uncaring & abnormal & abnormal & Borderline \\
\cline { 2 - 6 } & unemotional & borderline & borderline & $\begin{array}{l}\text { Abnormal } \\
\text { Borderline }\end{array}$ \\
\hline
\end{tabular}

Table 5. Family influence in ICU and externalizing SDQ association

\begin{tabular}{|l|l|l|l|l|l|}
\hline \multicolumn{2}{|c|}{} & & \multicolumn{3}{|l|}{ Externalizing SDQ } \\
\hline & Total & Divorced & United family \\
\hline \multirow{3}{*}{ ICU } & callousness & Normal & Abnormal & Normal \\
\cline { 2 - 6 } & uncaring & abnormal & Borderline & abnormal \\
\cline { 2 - 6 } & unemotional & borderline & Normal & borderline \\
\hline
\end{tabular}

Table 6. Gender influence in ICU and externalizing SDQ association

\begin{tabular}{|l|l|l|l|l|}
\hline \multicolumn{2}{|l|}{} & & Externalizing SDQ & \\
\hline & Total & Women & Men \\
\hline \multirow{4}{*}{ ICU } & callousness & Normal & borderline & Normal \\
\cline { 2 - 6 } & uncaring & abnormal & abnormal & abnormal \\
\cline { 2 - 6 } & unemotional & borderline & normal & borderline \\
\hline
\end{tabular}

Investigating the internalizing scale, we found the same qualitative association highlighted between ICU traits and the total SDQ scale (figure 2). Furthermore, the analysed data showed 
that also the association between ICU traits and the internalizing behaviours is influenced by gender, family situation and drug use.

\section{Conclusions}

Correspondence analysis is a technique that has allowed to describe qualitatively the deviation from complete independence between the psychopathic traits on emotion recognition (callousness, uncaring and unemotional) measured using the Inventory of CallousUnemotional Traits (ICU) and the behavioral problems of adolescents (normal, borderline and abnormal) measured using Strengths and Difficulties Questionnaire (SDQ). Graphical presentation of the initial results of the survey gives the relationship between the analysed variables, which is a good starting point for an in-depth interpretation of the results. This technique significantly simplifies the interpretation of correlation between ICU and SDQ, giving information on close correlation between $\mathrm{CU}$ traits and mental disorders in adolescents.

Part of our findings confirms what has been previously found in literature: CU traits can be found in scholar age too, are prevalent in males and are more correlated to externalizing behaviours (Garcia et al., 2018 and the interesting findings of Raschle at al., 2018). The analysis of the single traits gave us interesting and unexpected results instead: the callousness has been related to a normal SDQ while alarming results have been found in relation to the uncaring and the unemotional feature going against a line of research that put in doubt the utility of the assessment of the unemotional feature to detect the real presence of the callous-unemotional (Moore et al., 2017). The unemotional feature is principally correlated with borderline and abnormal SDQ scores and this can mean that individuals with just the callousness trait prevalent, could be abler to camouflage behavioral outcomes too.

The gender influence is resulted relevant in the evaluation of the externalizing and internalizing scales but not in the complete scale of the SDQ. This evidence could also reflect precedent findings: boys and girls have different ways to manifest $\mathrm{CU}$ traits as aggressiveness (Eme, 2016). This hypothesis finds confirmation, for example, if we consider bullying acts, that are done more directly by males than females. The callousness trait, that in males is related to a normal SDQ, in females is correlated to and abnormal dimension, when analysing the externalizing and internalizing scales.

Other variables that influence the manifestation of problem/behaviour relate to CU traits are the familiar situation and the use of drugs. The family situation is a variable that the young individual usually endures and passively accepts and thus can create interior suffering and different form of conflict. At in early age, in which the mechanism of the identity construction is active, a stable home situation is a fundamental to not make that conflict, and the emotion underlying it, going out of control.

The use of drugs can be related to familiar conflicts too and exacerbate mental and behavioral outcomes, compromising the brain functioning long-life. Hence, it is not surprising that individuals who assume drugs show a worsening of the manifestation of the callousunemotional. Those relations have been found in all our analysis (the relation between the complete and the partial scales of the SDQ and the CU traits). 
There is an increasing number of studies that suggests possible parallelism between callousunemotional traits and other emotional deficiencies that require special needs, such as those related to autism (Georgiou et al., 2019). We are dealing with young people unable to read their emotional code, with judgement difficulties and unable to process negative feedbacks properly. It is possible to affirm that those adolescents are not able to read their emotions. They never learned (because they weren't taught it?) to link concepts to their feelings, thus they are not able to create cognitive connections in order to express themselves. They cannot count on an emotional code sufficient to read reality in objective terms.

It could be hypothesised that even for the development of callous-unemotional traits the early relation with the caregiver has a fundamental role (Goulter et al., 2017; Dadds et al., 2019). This relation influences the psycho-emotional and educational development of the subject. Hence, from a pedagogical perspective, the possibility to associate these traits to behavioral problems and to personal situation (gender, familiy's status and use of drugs) it is fundamental to early detection of the psychopathy, thus allowing the use of pedagogical strategies for young people who have a high risk of severe conduct disorders.

\section{Acknowledgements}

We thank the principals, the professors and the students who collaborated on the project.

\section{References}

Agresti, A. (2002) Categorial Data Analysis. New York: Wiley.

American Psychiatric Association (2013). Diagnostic and Statistical Manual of Mental Disorders (5th ed.), Author, Washington, DC.

Beauchaine \& S. P. Hinshaw (Eds.), Oxford library of psychology. The Oxford handbook of externalizing spectrum disorders (p. 239-263). Oxford University Press.

Beh, E. J. (2004). Simple Correspondence Analysis: A Bibliographic Review. International Statistical Review, 72(2), 257-284.

Bendixen M. (2003). A Practical Guide to the Use of Correspondence Analysis in Marketing Research. Marketing Bulletin, 14, 16-38.

Benesch, C., Görtz-Dorten, A., Breuer, D., Döpfner, M. (2014). Assessment of callousunemotional traits in 6 to 12 year-old children with Oppositional Defiant Disorder/Conduct Disorder by parent ratings. Journal of Psychopathological Behavior Assessessment, 36(4), 519-529.

Bergstrøm, H., \& Farrington, D. P. (2018). Grandiose-manipulative, callous-unemotional, and daring-impulsive: The prediction of psychopathic traits in adolescence and their outcomes in adulthood. Journal of Psychopathology and Behavioral Assessment, 40(2), 149-158.

Bishop S.A., Okagbue H.I., Odukoya J.A. (2020). Statistical analysis of childhood and early adolescent externalizing behaviors in a middle low income country. Heliyon, 6(2). 
Blair, R., J., R., Leibenluft, E., Pine, D.S. (2014). Conduct disorder and callous-unemotional traits in youth. The New England Journal of Medicine, 371, 2207-2216.

Dadds, M., R., English, T., Wimalaweera, S., Schollar-Root, O. and Hawes, D., J. (2019). Can reciprocated parent-child eye gaze and emotional engagement enhance treatment for children with conduct problems and callous-unemotional traits: a proof-of-concept trial. Journal of Child Psychology and Psychiatry, 60, 676-685. doi:10.1111/jcpp.13023

Eckart, C., Young, G. (1936). The approximation of one matrix by another of lower rank, Psychometrika, 1, 211-218.

Eme, R. (2016). Sex differences in the prevalence and expression of externalizing behavior. In T. P.

Ezpeleta, L., de la Osa, N., Granero, R., Penelo, E., Domènech, J., M. (2013). Inventory of callous-unemotional traits in a community sample of preschoolers. Journal of Clinical Child \& Adolescent Psychology, 42(1), 91-105.

Frick P.J. (2004). The Inventory of Callous-Unemotional traits: Unpublished rating scale.

Frick, P., J., Ellis, M. (1999). Callous-unemotional traits and subtypes of conduct disorder. Clinical Child and Family Psychology Review, 2(3), 149-68.

Garcia, A., M., Graziano, P., A. \& Hart, K., C. (2018). Response to Time-Out Among Preschoolers with Externalizing Behavior Problems: The Role of Callous-Unemotional Traits. Child Psychiatry Human Development, 49, 699-708. https://doi.org/10.1007/s10578-0180788-6

Georgiou, G., Demetriou, C., A., Fanti, K., A. (2019). Distinct Empathy Profiles in Callous Unemotional and Autistic Traits: Investigating Unique and Interactive Associations with Affective and Cognitive Empathy. Journal of Abnormal Child Psychology, 47, 1863-1873. https://doi.org/10.1007/s10802-019-00562-1

Goodman R. (1997). The Strengths and Difficulties Questionnaire: a research note. Journal of child psychology and psychiatry, and allied disciplines, 38(5):581-600.

Goodman R. (1999). The extended version of the Strengths and Difficulties Questionnaire as a guide to child psychiatric caseness and consequent burden. Journal of child psychology and psychiatry, and allied disciplines, 40(5):791-9.

Goodman. R. (2001). Psychometric properties of the strengths and difficulties questionnaire. Journal of the American Academy of Child and Adolescent Psychiatry, 40 (11), 1337-1345.

Greenacre M. (1984). Theory and Application of Correspondence Analysis. London: Academic Press

Greenacre M. and Hastie T. (1987). The Geometric Interpretation of Correspondence Analysis, Journal of the American Statistical Association, 82, No. 398, 437 - 447.

Goulter, N., Kimonis, E. R., Hawes, S. W., Stepp, S., \& Hipwell, A. E. (2017). Identifying 
stable variants of callous-unemotional traits: A longitudinal study of at-risk girls. Developmental Psychology, 53(12), 2364-2376. https://doi.org/10.1037/dev0000394

Hall C., Guo B., Valentine A., Groom M., Daley D., Sayal K., Hollis C. (2019). The validity of the Strengths and Difficulties Questionnaire (SDQ) for children with ADHD symptoms. US National Library of Medicine National Institutes of Health.

Henry, J., Pingault, J., B., Boivin, M., Rijsdijk, F., Vidinh, E. (2016). Genetic and environmental aetiology of the dimensions of Callous-Unemotional traits. Psychological Medicine, 46(2), 405-414.

Katsimicha, E. (2016). Can Callous-Unemotional Traits be Reliably Measured in Preschoolers? Journal of Abnormal Child Psychology, 44(4), 625-38.

Kessler R.C., Berglund P, Demler O, Jin R, Merikangas K.R., Walters E.E. (2005). Lifetime prevalence and age-of-onset distributions of dsm-iv disorders in the national comorbidity survey replication. Archives of General Psychiatry, 62(6):593-602.

Kilon J. and Dębkowska K. (2013). The use of correspondence analysis for delphi survey results interpretation, Quantitative methods in economics, 14(1), 222-231

Kimonis, E., R., Fanti, K., A., Anastassiou-Hadjicharalambous, X., Mertan, B., Goulter, N.,

Law, D., \& Wolpert, M. (2014). Guide to using outcomes and feedback tools with children, young people and families. UK: Press CAMHS.

Levy, T., Apter,A., Djalovski, A., Peskin,M., Fennig, S., et al. (2017). The reliability, concurrent validity and association with salivary oxytocin of the self-report version of the Inventory of Callous-Unemotional Traits in adolescents with conduct disorder. Psychiatry Research, 256, 124-129. doi.org/10.1016/j.psychres.2017.06.028.

Levy, T., Bloch, Y., Bar-Maisels, M., Gat-Yablonski, G., Djalovski, A., Borodkin, K., Apter. A. (2015). Salivary oxytocin in adolescents with conduct problems and Callous-Unemotional Traits. European Child and Adolescent Psychiatry, 24, 1543-1551.

Liu J., Chen X., Lewis G. (2011). Childhood internalizing behaviour: analysis and implications. J Psychiatr Ment Health Nurs, 18(10):884-894.

Lundh, L.G., Wangby-Lundh, M., \& Bjarehed, J. (2008). Self-reported emotional and behavioral problems in Swedish 14 to 15-year-old adolescents: A study with the self-report version of the Strengths and Difficulties Questionnaire. Scandinavian Journal of Psychology, 49:523-532.

Merikangas KR, He J-p, Burstein M, Swanson SA, Avenevoli S, Cui L, et al. (2010). Lifetime Prevalence of Mental Disorders in U.S. Adolescents: Results from the National Comorbidity Survey Replication-Adolescent Supplement (NCS-A). Journal of the American Academy of Child and Adolescent Psychiatry, 49(10):980-900.

Moore, A., A., Carney, D., Moroney, E., Machlin, L., Towbin, K., E., Brotman, M., A., et al. 
(2017). The Inventory of Callous-Unemotional Traits (ICU) in children: reliability and heritability. Behavioral Genetics, 47(2), 141-151. 10.1007/s10519-016-9831-1

Pearson, K. (1900). On a criterion that a given system of deviations from the probable in the case of a correlated system of variables is such that it can be reasonably supposed to have arisen from random sampling. Philosophical Magazine (Series 5), 50, 157-175.

Pearson, K. (1904). On the theory of contingency and its relation to association and normal correlations. Drapers Company Research Memoirs (Biometric Series), 1.

Pearson, K. (1906). On certain points connected with scale order in the case of a correlation of two characters for some arrangement give a linear regression line. Biometrika, 5, 176-178.

Polanczyk G.V., Salum G.A., Sugaya L.S., Caye A, Rohde L.A. (2015). Annual research review: A meta-analysis of the worldwide prevalence of mental disorders in children and adolescents. Journal of Child Psychology and Psychiatry, and allied disciplines, 56(3):345-65.

Raschle, N., M., Menks, W., M., Fehlbaum,L., V., Steppan, M., Smaragdi, A., GonzalezMadruga, K., Rogers, J., Clanton,R., Kohls, G. et al. (2018). Callous-unemotional traits and brain structure: Sex-specific effects in anterior insula of typically-developing youths. NeuroImage: Clinical, 17, 856-864.

Roy S. N. and Kastenbaum M.A. (1956). On the Hypothesis of No "Interaction" In a Multiway Contingency Table. Annals of Mathematical Statistics, 27, 749-757.

Viding, E., Simmonds, E., Petrides, K., V., Frederickson, N. (2009). The contribution of callous-unemotional traits and conduct problems to bullying in early adolescence. Journal of Child Psychology and Psychiatry, 50(4), 471-81.

Vostanis, P. (2006). Strengths and difficulties questionnaire: research and clinical applications.

Current Opinion in Psychiatry, 19, 367-372.

\section{Copyright Disclaimer}

Copyright reserved by the author(s).

This article is an open-access article distributed under the terms and conditions of the Creative Commons Attribution license (http://creativecommons.org/licenses/by/3.0/). 